\title{
Landschaftsveränderungen, kartographisch dargestell - Das Kartenpaar «Ronco» aus dem Atlas der Schweiz als Beispiel
}

\section{Grundsätzliches zur kartographischen Darstellung von Veränderungen}

Mit Karten können wir nicht die Wirklichkeit wiedergeben, sondern, wie es in einer aktuellen Definition der Kartographie heißt, «eine ganzheitliche Darstellung und intellektuelle Abstraktion der geographischen Realität, die für einen bestimmten Zweck weitervermittelt werden soll». Beim Großteil aller Karten bezieht sich diese geographische Realität auf einen ganz bestimmten Zeitpunkt, auf das Datum der Erhebungen, auf den Abschluß der Redaktionsarbeiten, den sogenannten Stand. Es vereinfacht für den Kartenbenützer das Verständnis der Karte, wenn er weiß, daß sich die dargestellte Information auf einen einheitlichen Stand bezieht. Typische Vertreter solcher Momentanbilder sich die topographischen Karten. Alle Details, die in ihnen enthalten sind, widerspiegeln die Zustände zu einem ganz bestimmten Datum. Bei modernen Kartenaufnahmen ist dies in der Regel das Datum der Flugaufnahmen.

Bei der Darstellung von Landschaftsveränderungen jedoch bekommt die Komponente Zeit eine ganz besondere Bedeutung. Sie wird zu einer Variablen des Themas. Ihr kann durch verschiedene Methoden Rechnung getragen werden. Das einfachste Verfahren besteht wohl darin, zwei oder mehrere Stände desselben Gebiets, mit gleichem Zeichenschlüssel bearbeitet, nebeneinanderzustellen (Abb. 1 und 2). Es bleibt dann dem Kartenleser überlassen, durch Vergleichen der verschiedenen Kartenbilder die mehr oder weniger großen Unterschiede herauszufinden und zu interpretieren. Das kann eine spannende Angelegenheit sein, vergleichbar dem Spiel auf der Rätselseite: «Wer findet am meisten Unterschiede?» Das Hauptproblem dieser Methode besteht darin, den Überblick über die gefundenen Unterschiede nicht zu verlieren, sie sich wirklich merken zu können.

Deshalb ist es naheliegend, diese Veränderungen direkt sichtbar zu machen, indem sie mit eigenen Kartenzeichen dargestellt werden. Mit den Abbildungen 3 bis 8 soll exemplarisch gezeigt werden, was für Möglichkeiten sich aus den beiden Abbildungen 1 und 2 ableiten lassen. Für solche Vergleiche wird man in konkreten Anwendungen allerdings die Landeskarte 1:25000 vorziehen, denn mit der Karte im Maßstab 1: 100000 gewinnt man wegen der Generalisierung nur ein summarisches Bild. Denken wir nur daran, daß in diesem Maßstab nur 30\% aller Häuser dargestellt werden können und auch das Straßen- und Wegnetz unvollständig ist. Zur Illustration des Verfahrens mag das vorliegende Beispiel, das sogenannte totale Bild eines Ausschnittes aus der Landeskarte 1:100000, jedoch genügen. Um die Details besser erkennbar zu machen, wurden alle Ausschnitte auf 1:50 000 vergrößert.

Die Abbildungen 3 und 4 sind Differenz- oder Veränderungsbilder. Sie gehen rein kopiertechnisch aus den Abbildungen 1 und 2 hervor: Man deckt das Negativ von Abb. 1 mit dem Positiv von Abb. 2 ab. Transparent bleiben damit nur noch diejenigen Elemente, die in der neuen Karte nicht mehr enthalten sind. Die Abb. 3 ist das Positiv, das von diesem «Sandwich» kopiert und noch geringfügig retouchiert wurde. Abb. 4 wiederum entsteht als Positiv aus dem Negativ der Abb. 2, überlagert mit dem Positiv von Abb. 1. Diese beiden Bilder können aber kaum für sich allein wiedergegeben werden, sondern machen ihre Einbettung in den ursprünglichen oder in den Endzustand notwendig. Beispielsweise können auf diese Weise vor dem Hintergrund des aktuellen Kartenbildes die Veränderungen gezeigt werden, die seit einem früheren Zustand eingetreten sind (Abb. 6). Das Veränderungsbild wird dabei als die wesentliche Aussage einer solchen Karte graphisch dominant zu gestalten sein. Das Hintergrundbild dient der räumlichen Einordnung der verschiedenen Veränderungen und wird bei der Interpretation auf der Suche nach Begründungen beigezogen. Aber auch die umgekehrte Version, früherer Zustand als Hintergrundbild mit aufgesetzten Veränderungen, kann zu einem sehr wirkungsvollen Resultat führen (Abb. 5). Diese Methode wird allerdings seltener angewandt, vor allem weil der frühere Zustand oft nicht mehr in einer direkt brauchbaren Form zur Verfügung steht.

Oft ist auch von Interesse, welche Objekte in der Zwischenzeit verschwunden sind. In Abb. 7 werden sie unter der heutigen Situation als graues Bild sichtbar gemacht. Schließlich wird in Abb. 8 ver-

Ernst Spiess, Prof., Dipl. Ing., Institut für Kartographie, ETH Hönggerberg, $\mathrm{CH}-8093$ Zürich 


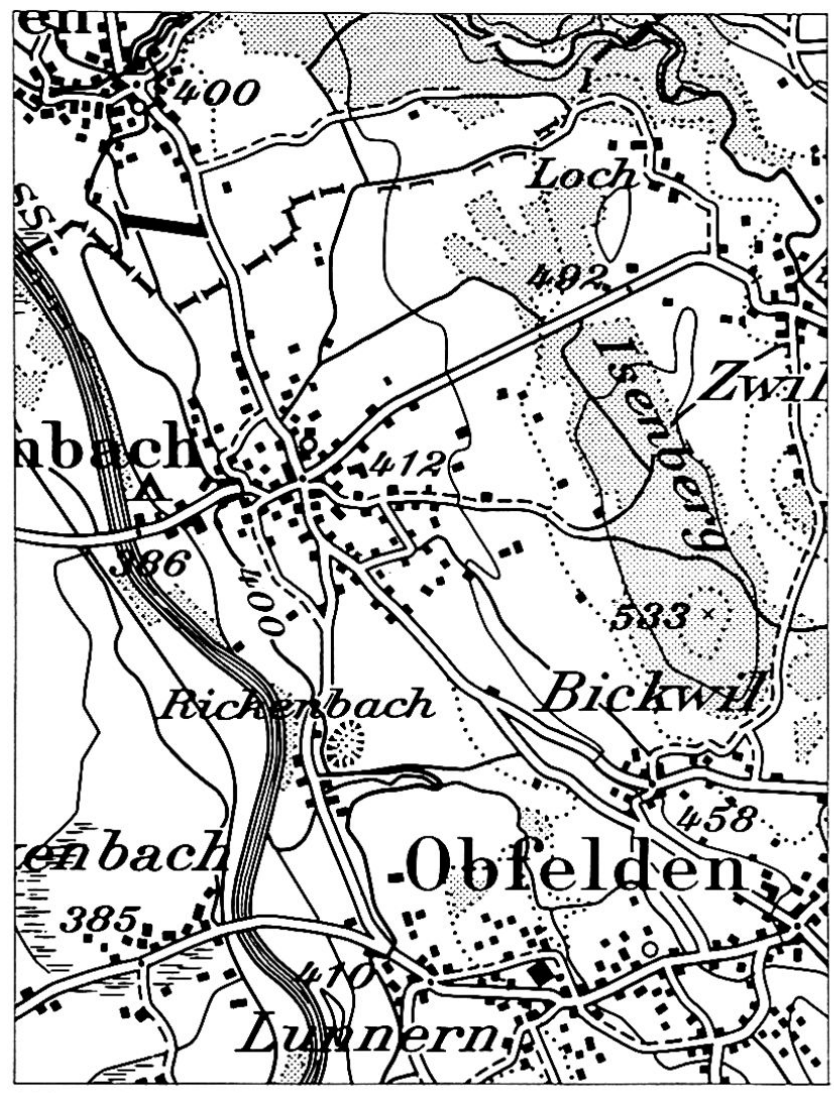

Abb.1 Ausschnitt aus der Landeskarte 1:100000, Ausgabe 1976, vergrössert auf 1:50000.

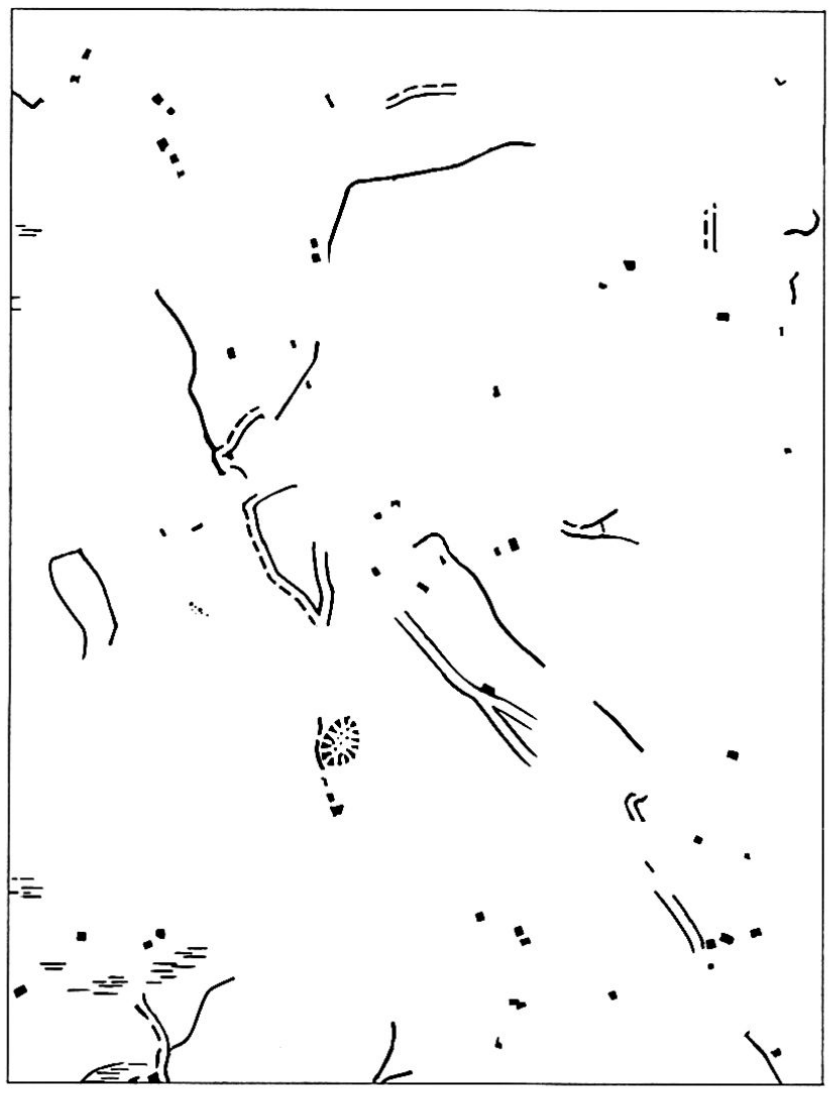

Abb.3 Das Differenzbild aus dem Negativ von Abb.1, abmaskiert mit dem Positiv von Abb.2, zeigt alle Elemente, die zwischen 1976 und 1982 verschwunden sind.

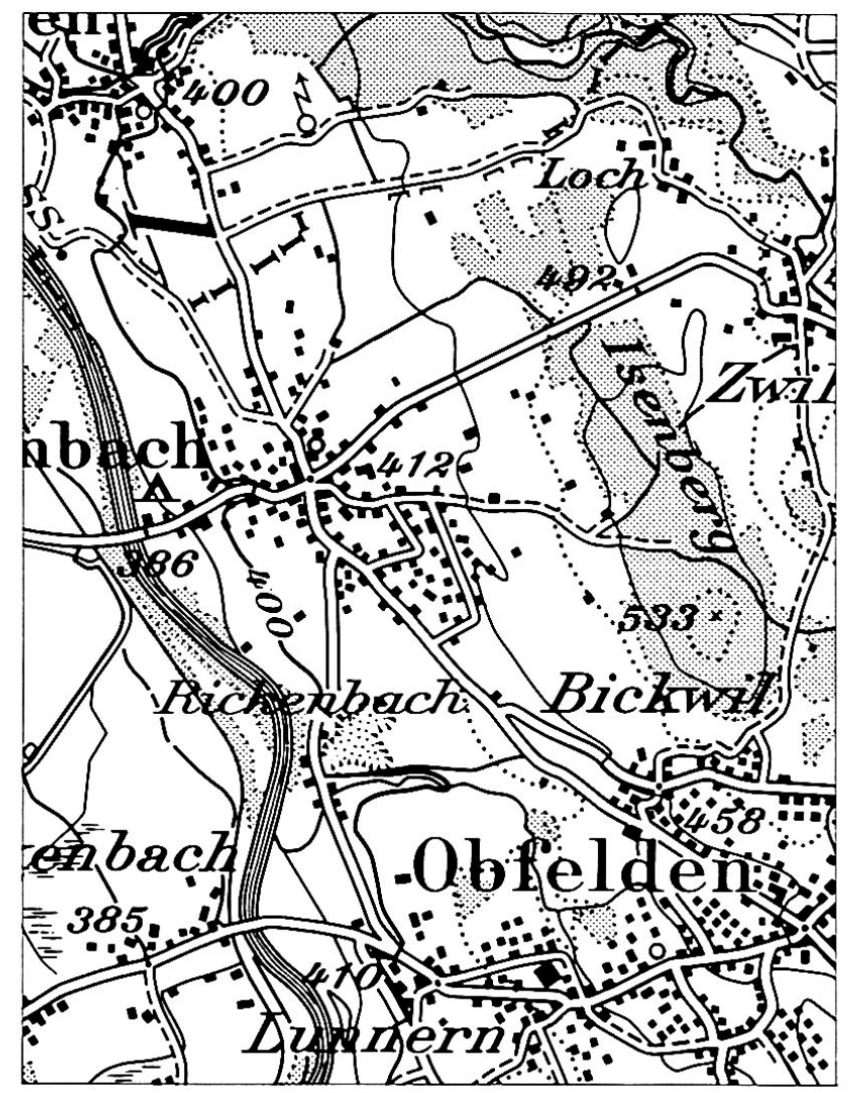

Abb.2 Derselbe Ausschnitt aus der Landeskarte 1:100000, Ausgabe 1982. Man versuche durch Vergleichen mit der Abb.1 alle Unterschiede festzuhalten.

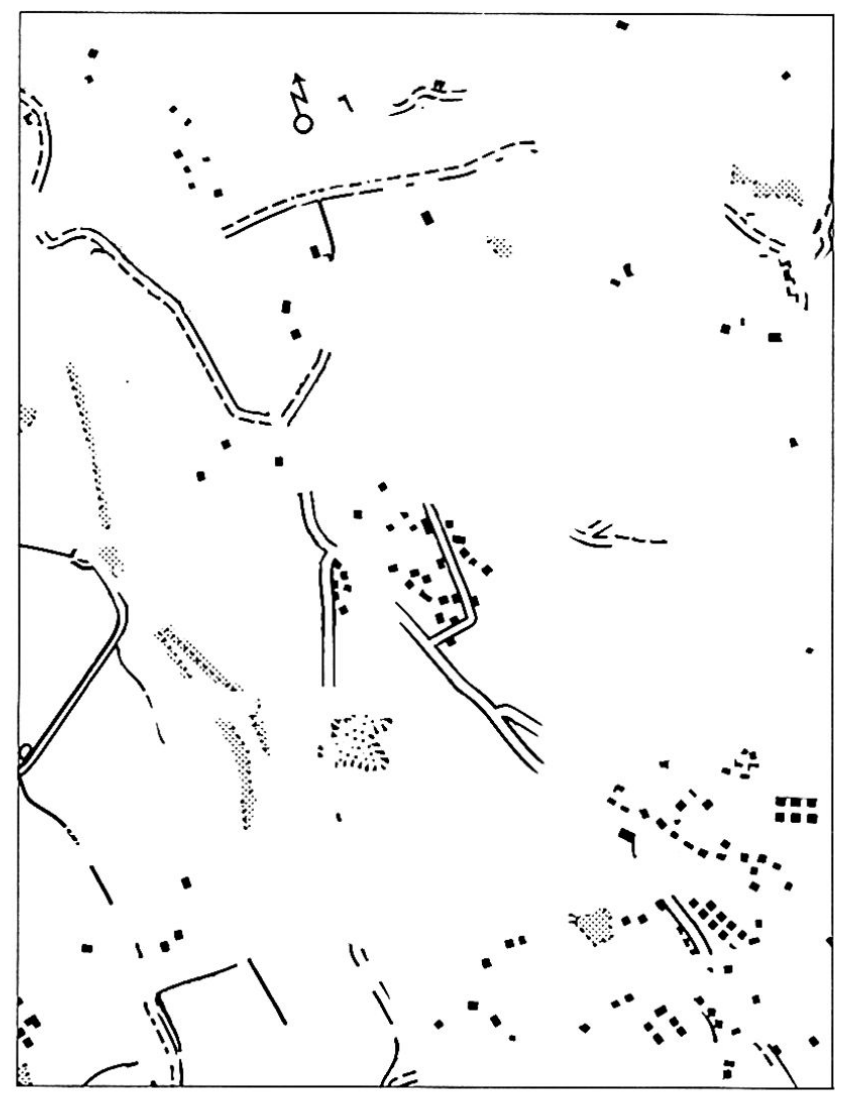

Abb.4 Das Differenzbild aus dem Negativ von Abb.2, abmaskiert mit dem Positiv von Abb.1, zeigt alle zwischen 1976 und 1982 neu hinzugekommenen Elemente.

Abb. 1 bis 8 und Kartenbeilage aus dem Atlas der Schweiz, reproduziert mit Bewilligung des Bundesamtes für Landestopographie vom 20. April 1990. 


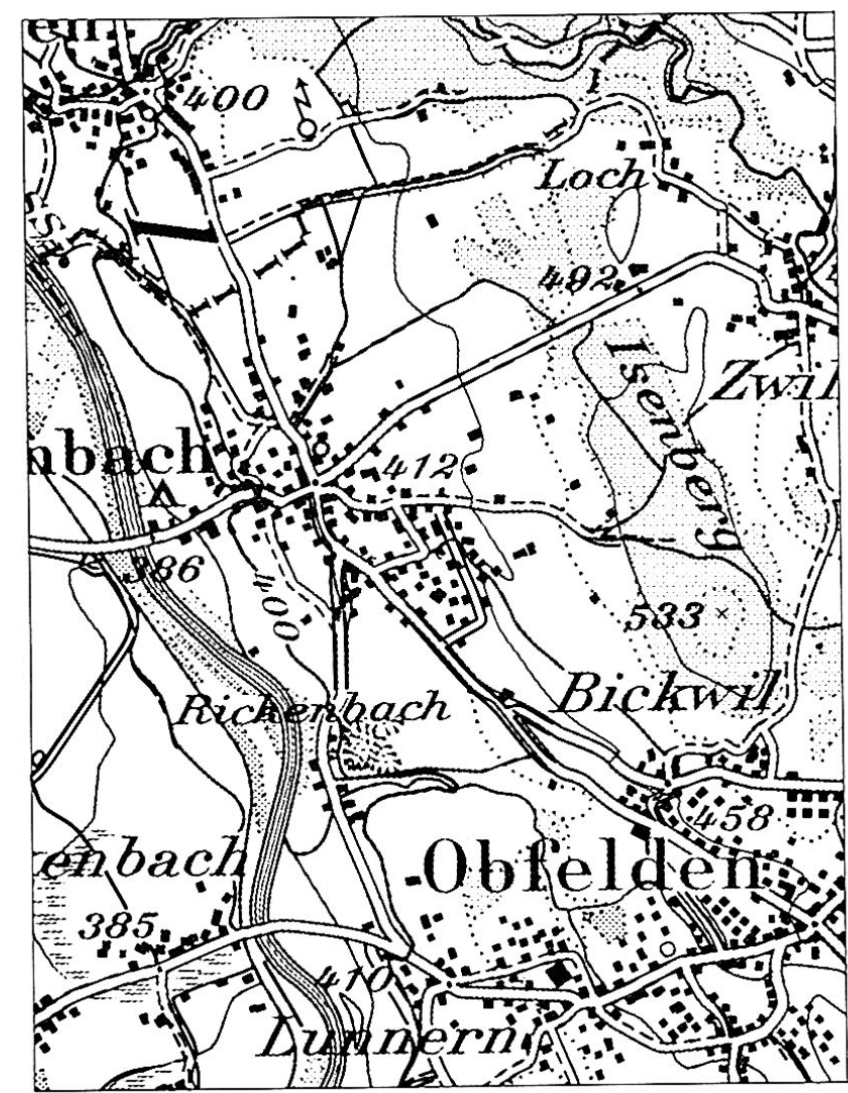

Abb.5 Landeskarte 1:100000; neue Elemente aus der Zeit zwischen 1976 und 1982 im vollen Schwarz auf dem grauen Hintergrund der Ausgabe von 1976.

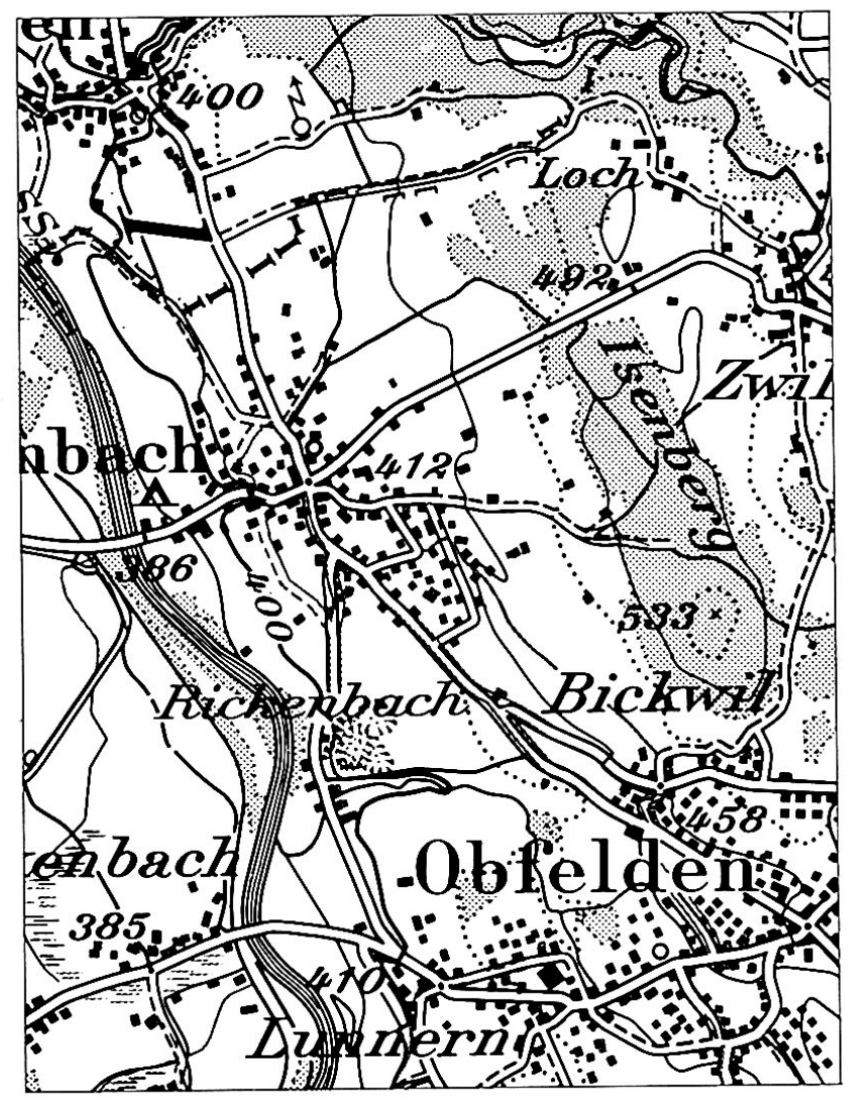

Abb.7 Landeskarte 1:100000; im Zeitraum 1976 bis 1982 verschwundene Elemente in grau unter der Ausgabe von 1982 im vollen Schwarz.

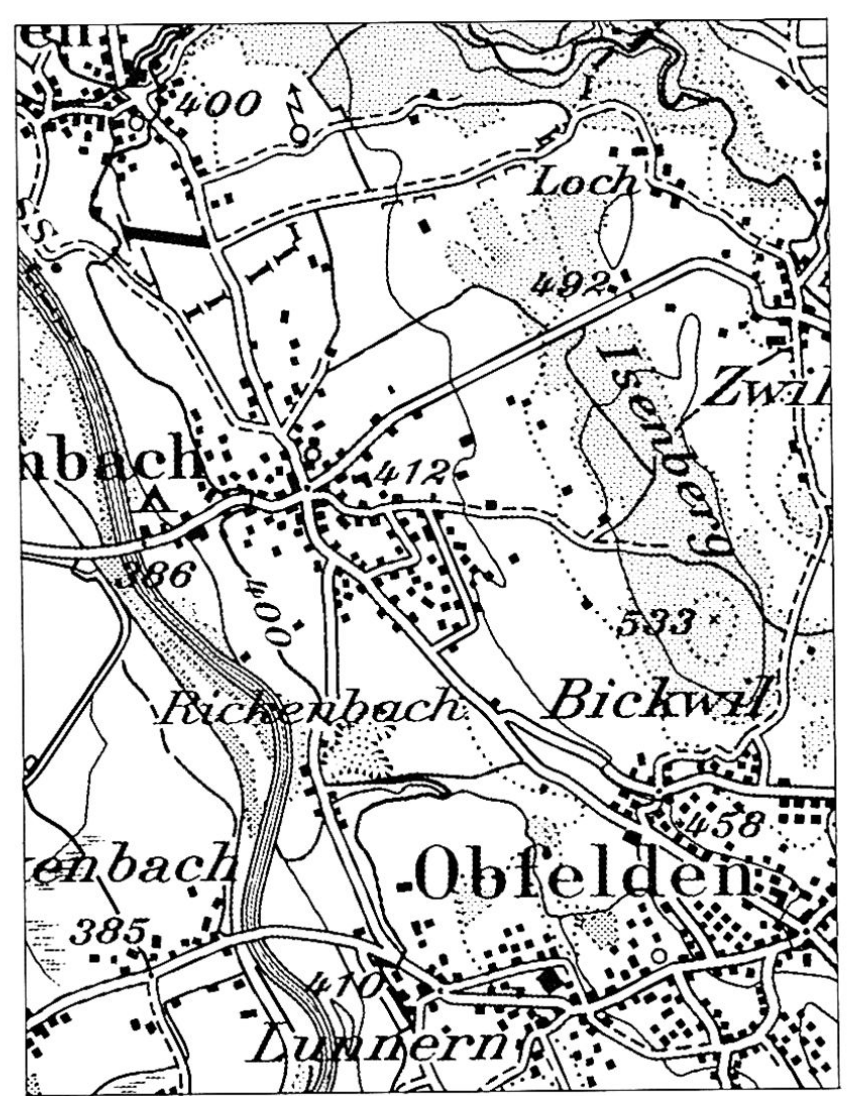

Abb. 6 Landeskarte 1:100000; neue Elemente aus der Zeit zwischen 1976 und 1982 im vollen Schwarz auf dem grauen Hintergrund der Ausgabe von 1982.

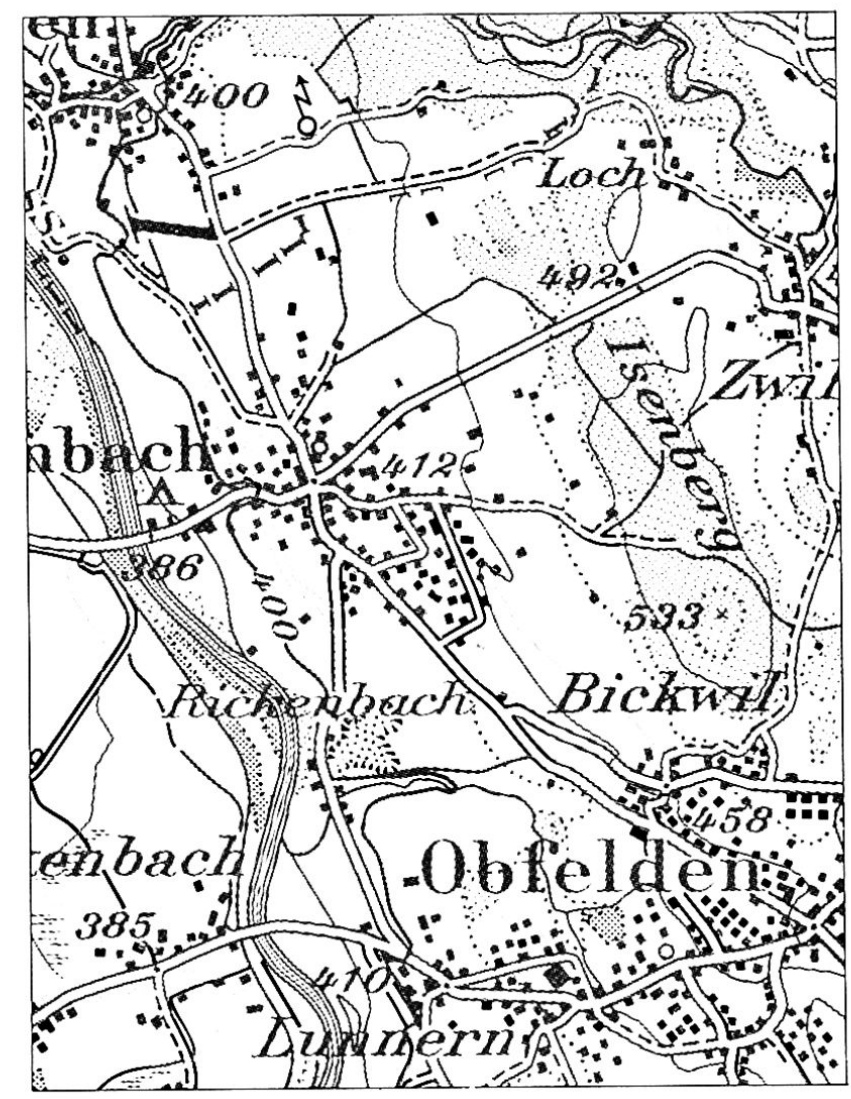

Abb.8 Landeskarte 1:100000; verschwundene Elemente in Grau, neue Elemente in Schwarz, auf der Grundlage der Ausgabe von 1982 (dunkelgrau). 
sucht, sowohl die verschwundenen Elemente (im Hellgrau) wie auch die neu entstandenen (im Schwarz) wiederzugeben.

Das einfarbige Kartenbild erschwert die Interpretation in verschiedener Hinsicht. Immerhin erkennt man, daß das frühere Hauptstraßennetz zwischen Ottenbach und Obfelden ausgebaut wurde und neue Quartierstraßen entstanden sind. Schwieriger zu erfassen ist der Ausbau von 4.-Klaß- zu 3.-KlaßStraßen. Im Gebiet von Rickenbach kommen im Zuge der Reußtalmelioration einige neue Waldstreifen dazu, die Kiesgrube hat sich stark erweitert, einige Kanäle wurden verlegt, was in der einfarbigen Karte besonders schwierig zu erkennen ist. Offensichtlich ist hingegen der Bau neuer Wohnquartiere in Obfelden und Ottenbach, wofür auch da und dort ältere Bausubstanz weichen mußte.

Die Verhältnisse komplizieren sich weiter, wenn nicht nur eine Zeitspanne behandelt wird, sondern die Veränderungen in mehreren Phasen darzustellen sind. Man wird in diesem Falle Prioritäten festlegen, also wichtigere Aspekte berücksichtigen und unwichtigere allenfalls unterdrücken müssen. Die Gestaltung einer solchen Karte ist in jedem Falle eine sehr anspruchsvolle Aufgabe. Die Objekte selbst sind durch unterschiedliche graphische Elemente charakterisiert, punktförmige, linienförmige und flächenförmige. Gleichartige Objekte sind zudem in verschiedenen Zuständen darzustellen, die den verschiedenen erfaßten Zeitpunkten entsprechen. Da diese eine klar geordnete Zeitreihe bilden, muß das graphische Darstellungsmittel eine ebensolche klare Ordnung widerspiegeln. Dazu kommen vor allem Hell-Dunkel-Skalen, kombiniert mit Farbe, in Frage. Probleme entstehen überall dort, wo sich solche Hell-Dunkel-Skalen überlagern. Nach der Helligkeit abgestufte Flächen, welche sich schneiden, zerstören sich gegenseitig ihre Reihenfolge und führen damit zu Mißverständnissen bei der Interpretation. Generell wird man versuchen, das Kartenbild aus drei Bildebenen aufzubauen. Im Idealfall sollten auf helle Flächentöne dunklere Linien und sehr kräftige Punktsignaturen aufgesetzt werden.

\section{Das Kartenpaar «Ronco sopra Ascona (TI)»}

Die Tafel 65 der 1 . Ausgabe des Atlas der Schweiz umfaßt eine Reihe von 7 Fremdenorten mit deren Infrastruktur und Siedlungscharakteristik. Der Stand dieser Erhebungen ist ca. 1971. Für die zweite Ausgabe dieser Tafel (65**) im Jahre 1981 entschlo $\beta$ sich die Redaktion, nicht einfach dieselben Detailkarten mit einem um 10 Jahre jüngeren Stand herauszugeben. Statt dessen wurden zwei neue Beispiele gewählt, nämlich Anzère, ein Walliser Wintersportort, der praktisch aus dem Nichts entstanden ist, und Ronco sopra Ascona, ein Tessiner Dorf, das viele Fremde angezogen und eine starke bauliche Entwicklung durchgemacht hat. In beiden Fällen wurde versucht, die Entwicklung dieser Gebiete in den letzten 50 Jahren nachzuzeichnen. Im folgenden befassen wir uns nur mit dem zweiten Fallbeispiel, das diesem Heft als farbige Beilage beigeheftet ist. Dabei beschränken wir uns zudem auf die landschaftliche Entwicklung, die durch die Stände von 1930 und 1980 charakterisiert ist, sowie auf vier Etappen der baulichen Entwicklung in diesem Zeitraum. Die Tafel $65^{* *}$ umfaßt außerdem eine Grundbesitzkartierung und eine Ortsplanung für dasselbe Gebiet, welche die geplante weitere Entwicklung dieser Gemeinde festlegt.

\section{Inhalt der Karte \\ «Ronco sopra Ascona (TI) im Jahre 1930»}

Teile dieser Karte, so z. B. die Höhenkurven, die Tiefenkurven im See, die Felszeichnung, die Namen und das Koordinatennetz, entstammen der Landeskarte $1: 25000$. Sie wurden für diese Darstellung auf das Doppelte, auf den Maßstab 1: 12 500, vergrößert. Mit Flächenfarbtönen werden einerseits die Seefläche des Langensees und anderseits die verschiedenen Kulturarten unterschieden. Den größten Flächenanteil nimmt der Buschwald ein, wobei mit einem gelben Farbton diejenigen Flächen noch unterschieden werden, die im Jahre 1930 teilweise abgeholzt waren. Als unproduktiv werden in Grau gewisse Felsflächen ausgeschieden. Das Kulturland in den tieferen Lagen ist mit einem bräunlichen Ton gekennzeichnet, darauf aufgesetzt in einer roten Rebensignatur - die hier übrigens nicht unbedingt dem natürlichen Aspekt entspricht - die Gebiete mit der typischen Tessiner Doppelkultur, Reben in Reihen mit Zwischenkulturen von Mais und Gemüse. Im Gebiet der Maiensäße (Monti) wird die offene Flur in Mähwiesen und Weiden unterteilt. Die großen, fruchttragenden Kastanienbäume sind mit kräftigen grünen Punkten eingetragen. Der Buschwald umfaßt natürlich ebenfalls vorwiegend Kastanienholz. In höheren Lagen kommen Birken, Eschen und Buchen vor, Nadelhölzer dagegen nur vereinzelt.

Die Häuser wurden hauptsächlich nach Wohngebäuden (schwarz), zeitweise bewohnten Hütten auf den Maiensäßen (hellbraun) und Ökonomiegebäuden oder Ställen (dunkelbraun) unterschieden. Zusätzlich wurden die 6 Hotels (blau) und die öffentlichen Gebäude wie Gemeindehaus, Kirche, Kapellen usw. (grau) gekennzeichnet. 16 zerfallene Häuser oder Ställe sind als Ruinen eingetragen. Als Sondersignaturen finden sich noch zahlreiche Bildstöcke zwischen dem Dorf Ronco und der Kapelle oberhalb Livurcio. Straßen, Saum- und Fußwege sind entsprechend der Legende der Landeskarte verzeichnet. 


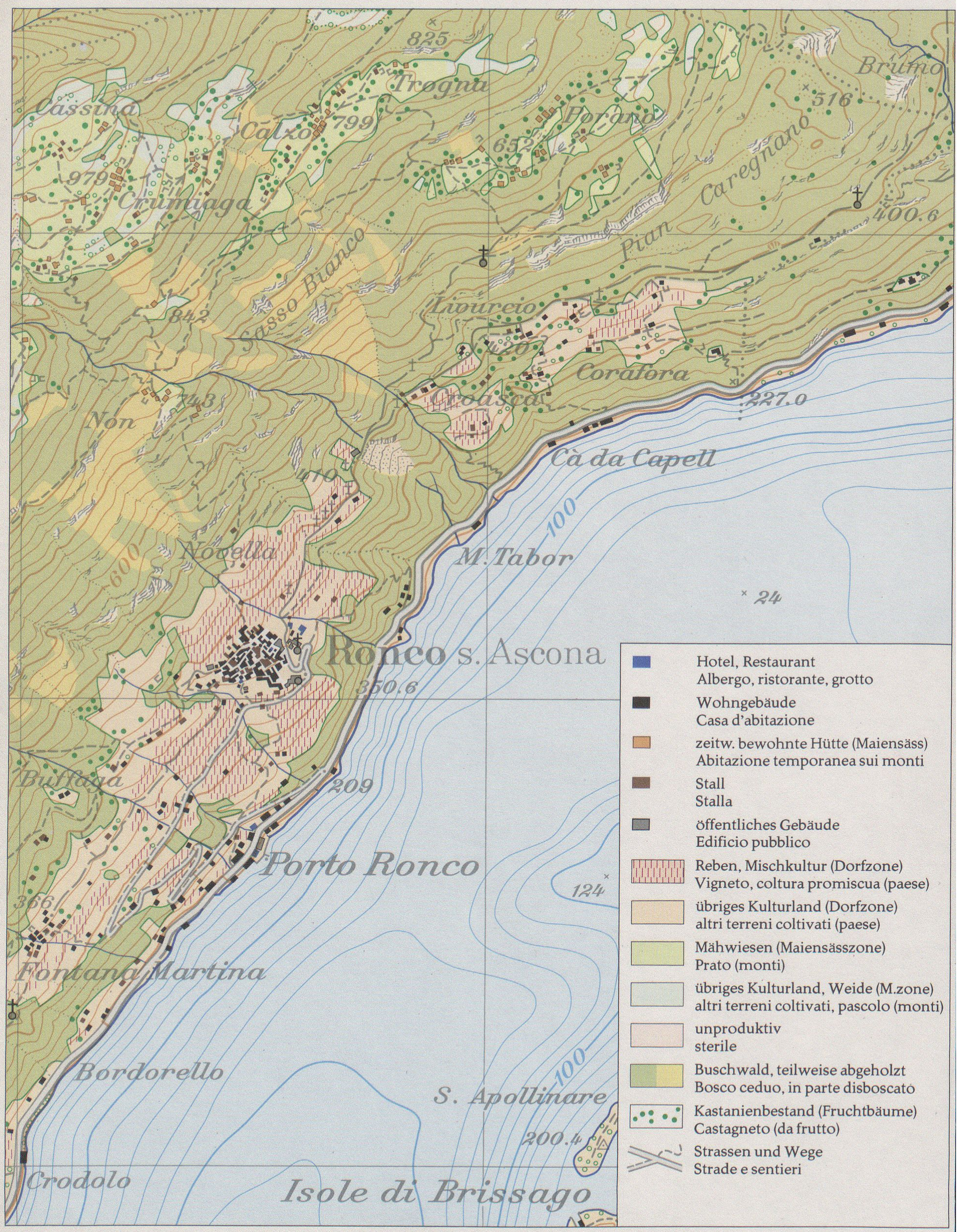

Ronco sopra Ascona (TI) im Jahre 1930

Ronco sopra Ascona (TI) nel 1930 


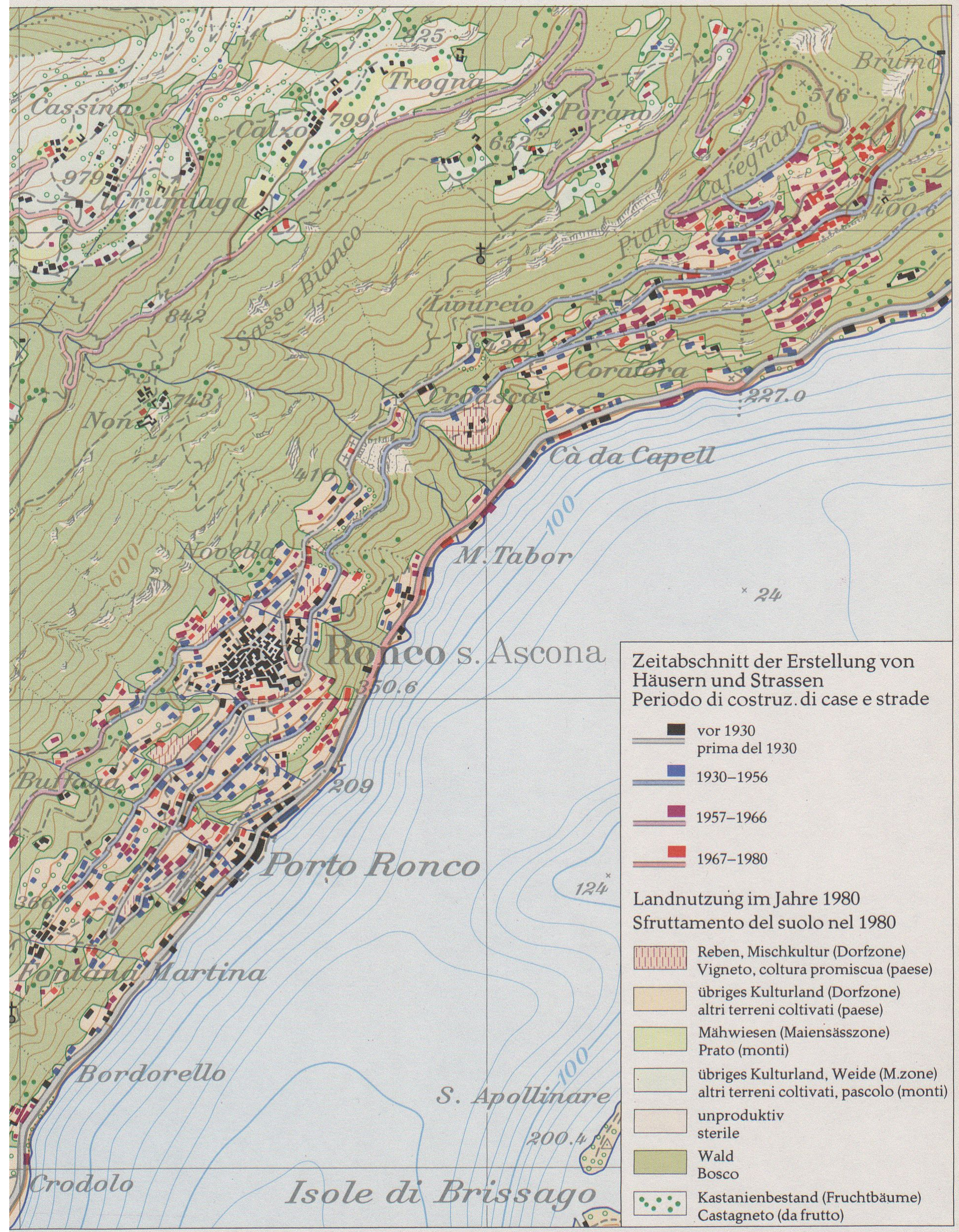

Ronco sopra Ascona im Jahre 1980, bauliche Entwicklung 1930-1980 Ronco sopra Ascona nel 1980, sviluppo edilizio dal 1930 al 1980 


\section{Inhalt der Karte \\ «Ronco sopra Ascona im Jahre 1980 . . .»}

Diese zweite Karte zeigt im wesentlichen den Stand von 1980, insbesondere was die Landnutzung anbetrifft. Der Zustand der baulichen Entwicklung von 1980 wird jedoch farblich in 4 Zeitphasen zerlegt: Schwarze Häuser und hellblaue Straßen waren schon 1930 vorhanden, blaue Häuser und dunkelblaue Straßen sind im Zeitraum 1930-1956 gebaut worden, rotviolette zwischen 1957 und 1966 und rote von 1967 bis 1980. Im Rahmen des darstellerisch Möglichen gibt diese farbliche Differenzierung deshalb ein Bild über den zeitlichen Ablauf der starken Bautätigkeit in Ronco. Die Hausfarbe hat in dieser Karte demnach eine andere Bedeutung als in der ersten Karte, wo sie Gebäudefunktionen unterscheidet. Auf die Darstellung der verschwundenen Bauten wurde dagegen verzichtet, dies mit Ausnahme derjenigen Häuser, von denen noch Überreste sichtbar sind.

Beim Kulturland wurden dieselben Kategorien wie in der Karte von 1930 beibehalten. Geändert wurde nur die Umschreibung des Waldes, da der heutige Wald mit demjenigen von 1930 nicht mehr direkt verglichen werden kann. Die gesamte Bestockung ist wesentlich dichter, was durch einen leicht dunkleren Farbton zum Ausdruck gebracht wurde.

\section{Quellen für diese Karten}

Für die Bearbeitung dieser Karten wurde das folgende Material zugezogen: In erster Linie wurde die Landeskarte 1:25000, Blatt Locarno, erste Ausgabe von 1962, sowie die weiteren Ausgaben von 1971 und 1977 als Grundlagen benützt. Das Gewässernetz und die Topographie samt Felszeichnung haben sich in den 50 Jahren nur minim verändert. Deshalb konnten diese Elemente für beide Karten praktisch ohne Änderungen übernommen werden. Zudem fand der «Piano corografico del Cantone di Ticino 1:5000», der Übersichtsplan, welcher teilweise die Grundlage für die neue Landeskarte bildete, Verwendung. Das Blatt 514, Locarno, des Siegfriedatlas, mit dem Maßstab $1: 50000$, aufgenommen 1895 und letztmals nachgeführt 1936, trägt kaum etwas zur Problematik bei, außer Hinweisen auf das damals benützte Straßenund Wegnetz. Dasselbe gilt für die erste Ausgabe der Landeskarte 1: 50000 von 1951.

Der Stand 1930 wurde praktisch ausschließlich aufgrund von Luftaufnahmen rekonstruiert, die seinerzeit für die Erstellung des oben genannten Übersichtsplans und der Landeskarte 1:50000 aufgenommen worden sind. Es handelt sich um Reihenbilder im Format $13 \times 13 \mathrm{~cm}$, die relativ tief geflogen worden sind und deshalb einen verhältnismäßig großen Bildmaßstab von 1:7000 aufweisen, was sich für unsere Zwecke als sehr günstig erwies. Ein Teil der Bilder waren als konvergente Bildpaare aufgenommen worden, d. h., die eine Aufnahme wurde senkrecht erstellt, die zweite vom selben Gebiet nach einiger Flugdistanz mit gekippter Kamera. Zum Zeitpunkt der Auswertung dieser Bilder für unsere Aufgabenstellung war kein Instrument mehr verfügbar, mit dem solche alte, konvergente Aufnahmen hätten ausgewertet werden können. Erst mit der Entwicklung der digitalen Photogrammetrie werden heute geometrisch genaue Auswertungen wieder möglich. Die Übertragung aller Befunde auf die Landeskarte $1: 25000$ erfolgte deshalb rein visuell. Es waren jedoch überall genügend Anhaltspunkte vorhanden, welche die Eintragung aller Einzelheiten erlaubten. Wo sich daraus Schwierigkeiten ergaben, wurden die Luftbilder, welche für die Nachführung der Landeskarte 1:25000 im Jahre 1977 erstellt worden waren, zum Vergleich herangezogen und für die Übertragung in den Grundriß als Zwischenprodukt benützt. Die Betrachtung unter dem Stereoskop erleichterte die Interpretation wesentlich, vor allem bei Hütten, im Siedlungsgebiet und im Wald, aber auch bei der Erfassung des Wegnetzes.

Neben diesem Bildmaterial wurden aber für die Bearbeitung des Kartenpaares zusätzlich noch andere Quellen benützt, vor allem die Publikation von GAL LUSSER (1968), sein Kommentar zu den Karten der Tafel $65^{* *}$ im Atlas der Schweiz und ein Planungsbericht von 1968. GALLUSSER hat sich, abgestützt auf seine Lokalkenntnisse, eingehend mit der Entwicklung in dieser Gemeinde auseinandergesetzt. Verschiedene seiner Erhebungen konnten in unser Kartenpaar übernommen oder eingearbeitet werden. Das gilt insbesondere für die erstellten Bauten in den verschiedenen Zeiträumen.

\section{Was den Karten nicht zu entnehmen ist}

Die erwähnten schriftlichen Quellen enthalten selbstredend auch eine größere Zahl von Hinweisen und Angaben, die aus der Karte nicht oder nur indirekt erkennbar wären. Als geländetypisches Merkmal fehlen z. B. sämtliche Mauern in der Landeskarte. Diese sind hingegen im Übersichtsplan 1: 5000 dargestellt. Die Vorgeschichte erwähnt den steten Rückgang der landwirtschaftlichen Nutzung des Gemeindegebiets seit ihrem Maximum zu Beginn des 19. Jahrhunderts und belegt diesen für die jüngste Periode mit folgenden Zahlen: 1930 waren noch 56 Beschäftigte in der Land- oder Forstwirtschaft tätig, 1970 zählte man nur noch 12 . Gleichzeitig stieg die Bevölkerung von 410 Einwohnern auf ca. 800 im Jahre 1974. Von letzteren sind rund ein Fünftel Ausländer und nur noch zwei Fünftel Einheimische. Die Zahl der Hotels und Pensionen ist bis 1966 auf 11 angewachsen. Zu deren 320 Betten 
kommen noch 600 in Ferienwohnungen und zeitweise bewohnten Villen dazu. Einen zahlenmäßigen Überblick über die bauliche Entwicklung gibt die folgende Tabelle der bewilligten Bauten:

$\begin{array}{rr}1930-1945 & 70 \\ 1946-1951 & 50 \\ 1952-1956 & 50 \\ 1957-1961 & 170 \\ 1962-1966 & 80 \\ 1967-1980 & 140\end{array}$

Es geht auch daraus hervor, da $\beta$ der eigentliche Bauboom 1955 durch den Bau der «Bürgergemeindestraße» nach Gruppaldo ausgelöst wurde, wo die Bürgergemeinde über größere Landflächen verfügte. Diese wurden parzelliert und an Interessenten, vorwiegend aus dem Ausland, verkauft. Erst die strengere Anwendung der Gesetzgebung über den Landschaftsschutz durch den Kanton und die Beschränkung der Verkäufe von Grundstücken an Ausländer durch den Bund haben, zusammen mit der Abschwächung der Konjunktur, die bauliche Entwicklung wieder verlangsamen können. Trotz der extremen Steilheit des Geländes wurden mehr als 20 Schwimmbassins gebaut. Sie sind aus maßstabähnlichen Gründen in der Karte nicht dargestellt.

Wenig können die Karten und die knapp gefaßten Legenden auch über die Nutzung des Waldes aussagen. Der größte Teil des Waldes, der insgesamt zwei Drittel der Gemeindefläche ausmacht, ist Niederwald und kann höchstens für Brennholz genutzt werden. Bis auf $800 \mathrm{~m}$ Höhe dominiert Kastanienunterholz. Darüber folgt ein Mischwald aus Birken, Kastanien und Eichen. Über $1000 \mathrm{~m}$ herrscht die Buche vor. Nadelholz ist eher selten. Was in der Karte nach neuen Walderschließungsstraßen aussieht, die interkommunale Straße Ronco-BuffagaBrissago und die Straße oberhalt Non, sind in Wirklichkeit Straßen, welche die Funktion von Brandschneisen haben. Schon der Verbindungsstraße nach Arcegno war ursprünglich diese Funktion zugedacht; sie wurde dann seinerzeit auch mit Subventionen der Forstorgane erbaut.

Diese Beispiele mögen genügen, um deutlich zu machen, daß ein schriftlicher Kommentar immer eine wertvolle, oft für deren Verständnis unentbehrliche Ergänzung zu einer Karte ist. Informationen, die nicht eine klare räumliche Strukturierung aufweisen und in wenige Klassen zusammengefaßt und gegliedert werden können, werden besser mit einem Text umschrieben.

\section{Vergleich der beiden Stände von 1930 und 1980}

Was lassen sich aus dem Vergleich dieser beiden Karten für Erkenntnisse gewinnen? Auffallend ist in erster Linie die starke Bautätigkeit in der Dorfzone unter $500 \mathrm{~m}$ ü. M. Sie hat mit Schwerpunkt im Gebiet zwischen Porto Ronco, Fontana Martina und im Bereich des Dorfes Ronco schon vor und direkt nach dem Zweiten Weltkrieg massiv eingesetzt. 172 Gebäude wurden allein in diesem Zeitraum erstellt. In diese Periode fällt auch der Ausbau der Erschließungsstraßen in Hangrichtung und der Verbindungsstraße nach Arcegno. Er bildet offensichtlich den Auslöser für die nächste Bauphase (rotviolette Häuser) mit eindeutigem Schwerpunkt im Gebiet von Gruppaldo unterhalb des Pian Caregnano, wo der Anteil ausländischer Grundeigentümer besonders hoch ist. In der letzten Phase (rote Häuser) stellt man schließlich eine gewisse Verdichtung in den meisten Baugebieten fest. In diesen Zeitraum fällt auch ein Teilausbau der Seeuferstraße und die Fertigstellung der Fahrstraße auf die Monti di Ronco. Anhand der Hausgrundrisse kann auch in gewissen Grenzen über den Ausbaustandard der einzelnen Bauten spekuliert werden. Große, unregelmäßige Grundrisse weisen auf Luxusvillen hin.

Die extreme Bautätigkeit hat sowohl das Kulturland wie auch den Wald stark zurückgedrängt. 1980 sind nur noch drei einigermaßen zusammenhängende, größere Rebanlagen übriggeblieben. Das übrige Kulturland in der Dorfzone ist weitgehend hypothetisch, da das Land rings um die neuen Wohnbauten kaum noch landwirtschaftlich genutzt wird.

Was den Wald anbetrifft, so stellt man beim genauen Vergleich zwei Erscheinungen fest. Einerseits wurde der Wald verschiedenenorts durch die Bautätigkeit massiv angeschlagen, so direkt über der Seeuferstraße und im ganzen Gebiet von Gruppaldo und Caregnano. Anderseits hat sich der Wald auf verschiedene Gebiete der früheren Anbauterrassen ausgedehnt, vor allem oberhalb des Dorfes. Einschränkend ist vielleicht zu bemerken, daß die Bestimmung der locker bestockten Waldfläche von 1930 aufgrund der Luftbilder mit einiger Unsicherheit behaftet ist. Generell ist der Buschwald in diesen 50 Jahren wesentlich dichter und hochstämmiger geworden. Der Grund dafür dürfte darin liegen, $\mathrm{da} ß$ heute weniger $\mathrm{Holz}$ geschlagen wird und der Wald nicht mehr mit Ziegen beweidet wird, wie noch vor dem Zweiten Weltkrieg. Die Kastanienselven mit den großen fruchttragenden Bäumen sind deutlich geschwunden. Das könnte zum Teil auf die Abgänge zurückgeführt werden, welche durch den Befall der Kastanienbäume durch den Rindenkrebs begründet sind.

Auf den Monti di Ronco stellt man einen starken Rückgang der Mähwiesen fest. Waren 1930 die Maiensäße fast durchwegs noch intensiv bewirtschaftet, so sind es 1980 nur noch einige wenige. Der größte Teil der offenen Flur auf den Monti ist heute Weideland oder mit Farnkraut überwuchertes Brachland, das vielleicht noch als Streue dient. Die Beweidung der Wälder hat oberhalb von Cassina, 
Calzo und Trogna zu einem deutlichen Schwund an geschlossener Waldfläche geführt.

Auf einige Sonderfälle wäre noch hinzuweisen. Das frühere Monti Non ist heute fast völlig zerfallen. Eine große Villa, die dort oben, weit über dem Dorf, gebaut worden war, wurde ein Raub der Flammen. Verschiedene Ställe und Hütten auf den Monti sind in den letzten Jahrzehnten zu einfachen Ferienhäusern ausgebaut worden. Neu errichtet wurden auch einige wenige größere Ökonomiegebäude, welche zusammen mit der neuen Bergstraße eine rationellere Bewirtschaftung der Maiensäße ermöglichen sollen. In Anlehnung an diese neue Straße sind auch einige Neubauten entstanden, wozu es manchenorts offenbar einer recht großzügigen Auslegung des Waldrodungsverbots bedurfte. $\mathrm{Ob}$ anderswo zur Kompensation aufgeforstet wurde, müßte von Fall zu Fall abgeklärt werden. Gesamthaft betrachtet scheint die Waldfläche doch deutlich vermindert worden zu sein.

\section{Schlußbemerkungen}

Von wenigen Ausnahmen abgesehen, können alle diese Beobachtungen aufgrund der beiden Karten angestellt werden. Diese Interpretation mag exemplarisch vor Augen führen, was für Schlüsse über die Landschaftsveränderungen sich aus dem Vergleich von Kartenmaterial ziehen lassen. Über die Jahre betrachtet, handelt es sich vielleicht um schleichende Veränderungen. Wählt man aber einen genügend großen Zeitraum, so wird der drastische Wandel und sein grundlegender Einfluß auf das Bild der Landschaft offensichtlich und eindrücklich. Solche Fallbeispiele stimmen nachdenklich und sollten auch der jungen Generation eine konkrete Vorstellung darüber verschaffen, was für eine Eigendynamik eingeleitete Prozesse entwickeln können. Zur Erklärung solcher Vorgänge, zu ihrer Begründung und zu ihrem besseren Verständnis bedarf es idealerweise zusätzlicher textlicher Erläuterungen. Aber bereits der Vergleich solcher Kartenbilder kann wesentliche Erkenntnisse vermitteln, verbunden mit der nicht unwichtigen Tatsache, daß darin das ganze Gebiet in homogener Weise bearbeitet ist.

Die Darstellung von Landschaftsveränderungen im und mit dem Kartenbild hat sich in neuerer Zeit zu einer wichtigen Methode zur Veranschaulichung der Auswirkungen von verschiedensten Entwicklungsprojekten entwickelt. So hat EWALD (1978) mehrere Landeskartenblätter bearbeitet und publiziert. Mehrere inhaltlich und graphisch unterschiedliche Beispiele finden sich auch im Schweizer Weltatlas (Zürich, Sarganserland, Venogemündung, Roseg- und Tschiervagletscher). Sie werden in der Neuausgabe noch um einige Kartenpaare ergänzt werden. Aber auch für konkrete Planungs- und Projektierungsarbeiten sind zum Teil umfangreiche Kartierungen im Gange, erwähnen wir nur die Rekonstruktion früherer Fluß- und Bachläufe. Im Rahmen des kürzlich angelaufenen NF-Projekts Kulturlandschaftswandel soll am Beispiel einer Gemeinde exemplarisch eine umfassende Methodologie zur Erfassung und Analyse von Veränderungen entwickelt werden. Graphische Komponenten werden auch dazu einen wichtigen Beitrag leisten können. Mit dem vorliegenden Beitrag möchte der Autor zu weitern solchen Interpretationen und einfachen Kartierungen anregen. Sie lassen sich praktisch auf jeder Schulstufe durchführen, fördern implizite auch das Kartenlesen und schärfen den Blick für solche zeitliche Veränderungen und ihre langfristigen Auswirkungen auf eine Region.

\section{Literaturhinweise}

GALLUSSER, W. A. (1968): Siedlungsentwicklung und Grundbesitzverhältnisse in der modernen Tessiner Kulturlandschaft, in Regio Basilensis IX/1, Basel 1968, S. 215-235, $10 \mathrm{Abb}$.

GALLUSSER, W. A. (1981): Kommentar zur Karte Ronco sopra Ascona (TI) der Tafel $65^{\star \star}$ des Atlas der Schweiz, Bundesamt für Landestopographie, Wabern-Bern, 2. Ausg. 1981.

NYDEGGER, A., et al. (1968): Rapporto di pianificazione locale: Ronco s. Ascona, SPF Zurigo 1968, 179 p., 11 ill.

EWALD, K. (1978): Der Landschaftswandel - zur Veränderung schweizerischer Kulturlandschaften im 20. Jahrhundert. Bericht Nr. 91, Eidg. Anstalt für das forstliche Versuchswesen, Birmensdorf 1978, S. 55-308, 14 Karten.

SPIESS, E. (1981): Schweizer Weltatlas, Ausg. 1981. Herausg. Konf. der Kant. Erziehungsdirektoren, Zürich 1981, 148 Kartenseiten. 